\title{
Trends in Self-reported and Biochemically Tested Marijuana Use Among Pregnant Females in California From 2009-2016
}

\author{
Kelly C. Young-Wolff, PhD, MPH, Lue-Yen Tucker, BA, Stacey Alexeeff, PhD, Mary Anne \\ Armstrong, MA, Amy Conway, MPH, Constance Weisner, DrPH, and Nancy Goler, MD \\ Division of Research, Kaiser Permanente Northern California, Oakland (Young-Wolff, Tucker, \\ Alexeeff, Armstrong); Early Start Program, Kaiser Permanente Northern California, Oakland \\ (Conway); Department of Psychiatry, University of California, San Francisco (Weisner); Regional \\ Offices, Kaiser Permanente Northern California, Oakland (Goler)
}

\begin{abstract}
Marijuana is the most commonly used illicit drug during pregnancy, and its use is increasing. From 2002 to 2014, the prevalence of self-reported, past-month marijuana use among US adult pregnant women increased from 2.4\% to 3.9\%. ${ }^{1}$ In aggregated 2002-2012 data, 14.6\% of US pregnant adolescents reported past-month use. ${ }^{2}$ However, studies are limited to selfreported surveys and likely underestimate use due to social desirability bias and underreporting, leaving the scope of the problem unclear. We investigated trends of prenatal marijuana use from 2009-2016 using data from a large California health care system with universal screening via self-report and urine toxicology.
\end{abstract}

\section{Methods}

The Kaiser Permanente Northern California (KPNC) institutional review board approved and waived consent for this study. KPNC is an integrated health care system serving approximately 4 million patients representative of the geographic area. The sample comprised KPNC pregnant females 12 years or older who completed a self-administered questionnaire on marijuana use since pregnancy and a cannabis toxicology test $(58 \%$ occurred at the same visit as the questionnaire, $87 \%$ occurred within 2 weeks of completing the questionnaire) during standard prenatal care (at approximately 8 weeks' gestation) from 2009 through 2016.

Corresponding Author: Kelly C. Young-Wolff, PhD, MPH, Division of Research, Kaiser Permanente Northern California, 2000 Broadway, Oakland, CA 94612 (kelly.c.young-wolff@kp.org).

Conflict of Interest Disclosures: All authors have completed and submitted the ICMJE Form for Disclosure of Potential Conflicts of Interest and none were reported.

Author Contributions: Dr Young-Wolff had full access to all of the data in the study and takes responsibility for the integrity of the data and the accuracy of the data analysis.

Concept and design: Young-Wolff, Tucker, Armstrong, Conway, Weisner, Goler.

Acquisition, analysis, or interpretation of data: Young-Wolff, Tucker, Alexeeff, Armstrong, Weisner, Goler.

Drafting of the manuscript: Young-Wolff.

Critical revision of the manuscript for important intellectual content: All authors.

Statistical analysis: Young-Wolff, Tucker, Alexeeff, Armstrong.

Obtained funding: Young-Wolff, Armstrong.

Administrative, technical, or material support: Young-Wolff, Tucker, Conway.

Supervision: Young-Wolff, Weisner, Goler. 
We estimated the adjusted prevalence of prenatal marijuana use via self-report or toxicology annually using Poisson regression with a log link function, controlling for age, race/ ethnicity, and median neighborhood household income using SAS (SAS Institute), version 9.3. Adjusted prevalence estimates used the average covariate distributions across the study period. We tested for linear trends and differences in trends by age. Two-sided $P$ values less than .05were considered statistically significant.

\section{Results}

Of 318085 pregnant females, 38628 (12.1\%) were excluded (1123 who missed the selfreported screening questionnaire [0.4\%], 37303 who missed a toxicology test [11.7\%], and 202 who missed both [0.06\%]). The race/ethnicity of the 279457 females (87.9\%) included in the study was $36.0 \%$ white, $27.9 \%$ Hispanic, $16.6 \%$ Asian, $5.9 \%$ black, and $13.6 \%$ other. The age ranges of the sample included females aged 12 to 17 years (1.4\%), 18 to 24 years (15.8\%), 25 to 34 years $(61.6 \%)$, and more than 34 years $(21.2 \%)$. The median neighborhood household income was $\$ 70677$ (interquartile range, $\$ 51$ 645-\$92 917).

From 2009 through 2016, the adjusted prevalence of prenatal marijuana use based on selfreport or toxicology increased from $4.2 \%$ (95\% CI, 4.0\%-4.5\%) to 7.1\% (95\% CI, 6.7\%$7.5 \%$ ) and was higher based on toxicology than self-report each year (Figure 1). Linear trend tests found use increased at an annual rate of 1.075 for self-report or toxicology (95\% CI, $1.064-1.085 ; P<.001), 1.083$ for toxicology (95\% CI, 1.072-1.094; $P<.001$ ), and 1.062 for self-report (95\% CI, 1.048-1.075; $P<.001)$.

The adjusted prevalence based on self-report or toxicology increased significantly from 2009 to 2016 for each age group (Figure 2). Use among females younger than 18 years to age 24 years increased the most, from $12.5 \%$ (95\% CI, $10.3 \%-14.7 \%$ ) to $21.8 \%$ (95\% CI, $16.5 \%-$ $27.2 \%$ ) for those younger than 18 years and from $9.8 \%$ (95\% CI, 9.0\%- 10.6\%) to $19.0 \%$ ( $95 \%$ CI, $17.8 \%-20.2 \%$ ) for those aged 18 to 24 years. Use among women aged 25 to 34 years increased from $3.4 \%$ (95\% CI, 3.1\%-3.7\%) to 5.1\% (95\% CI, $4.7 \%-5.4 \%$ ) and use among women older than 34 years increased from $2.1 \%$ (95\% CI, $1.7 \%-2.5 \%$ ) to $3.3 \%$ (95\% CI, 2.9\%-3.7\%). Linear trend tests found prenatal use increased at an annual relative rate of 1.088 for females younger than 18 years (95\% CI, 1.037-1.142; $P=.01$ ), 1.092 for those aged 18 to 24 years (95\% CI, 1.076-1.108; $P<.001), 1.080$ for those aged 25 to 34 (95\% CI, 1.047-1.113; $P<.001$ ), and 1.057 for those older than 34 years (95\% CI, 1.042 $1.072 ; P<.001)$. Trends varied by age $(P=.02)$.

\section{Discussion}

From 2009 to 2016, marijuana use among KPNC pregnant females increased from $4 \%$ to $7 \%$. Of concern, $22 \%$ of pregnant females younger than 18 years and $19 \%$ of pregnant females aged 18 to 24 years screened positive for marijuana use in 2016. Age-specific, selfreported prevalences were similar to US data, ${ }^{1}$ but toxicology prevalences were higher, suggesting use has been underestimated in self-reported surveys. In California, medical marijuana was legalized in 1996, and prenatal use may further escalate in 2018 when recreational marijuana is available legally. 
This study was limited to KPNC pregnant women screened for marijuana use at approximately 8 weeks' gestation. Prenatal use before vs after women realized they were pregnant could not be distinguished. Marijuana is detectable approximately 30 days after last use and varies with heaviness of use and marijuana potency. It is possible, but unlikely, that some toxicology tests identified prepregnancy use.

Initial evidence suggests that prenatal marijuana may impair fetal growth and neurodevelopment, ${ }^{3}$ but $79 \%$ of 785 pregnant women surveyed between 2007 and 2012 reported perceiving little to no harm in prenatal use. ${ }^{4}$ Continued monitoring of trends, exposure timing, and offspring outcomes is important as marijuana potency rises ${ }^{5}$ in an increasingly permissive legal landscape.

\section{Acknowledgments}

Funding/Support: This study was supported by a grant from the Kaiser Permanente Community Benefits Program and a NIDA K01 award (DA043604) from the National Institutes of Health.

Role of the Funder/Sponsor: The funders had no role in the design and conduct of the study; collection, management, analysis, and interpretation of the data; preparation, review, or approval of the manuscript; and decision to submit the manuscript for publication.

\section{References}

1. Brown QL, Sarvet AL, Shmulewitz D, Martins SS, Wall MM, Hasin DS. Trends in marijuana use among pregnant and nonpregnant reproductive-aged women, 2002-2014. JAMA. 2017; 317(2):207209. [PubMed: 27992619]

2. Salas-Wright CP, Vaughn MG, Ugalde J, Todic J. Substance use and teen pregnancy in the United States. Addict Behav. 2015; 45:218-225. [PubMed: 25706068]

3. Volkow ND, Compton WM, Wargo EM. The risks of marijuana use during pregnancy. JAMA. 2017; 317(2):129-130. [PubMed: 27992628]

4. Ko JY, Farr SL, Tong VT, Creanga AA, Callaghan WM. Prevalence and patterns of marijuana use among pregnant and nonpregnant women of reproductive age. Am J Obstet Gynecol. 2015; 213(2): 201e1-201.e10. [PubMed: 25772211]

5. ElSohly MA, Mehmedic Z, Foster S, Gon C, Chandra S, Church JC. Changes in cannabis potency over the last 2 decades (1995-2014). Biol Psychiatry. 2016; 79(7):613-619. [PubMed: 26903403] 


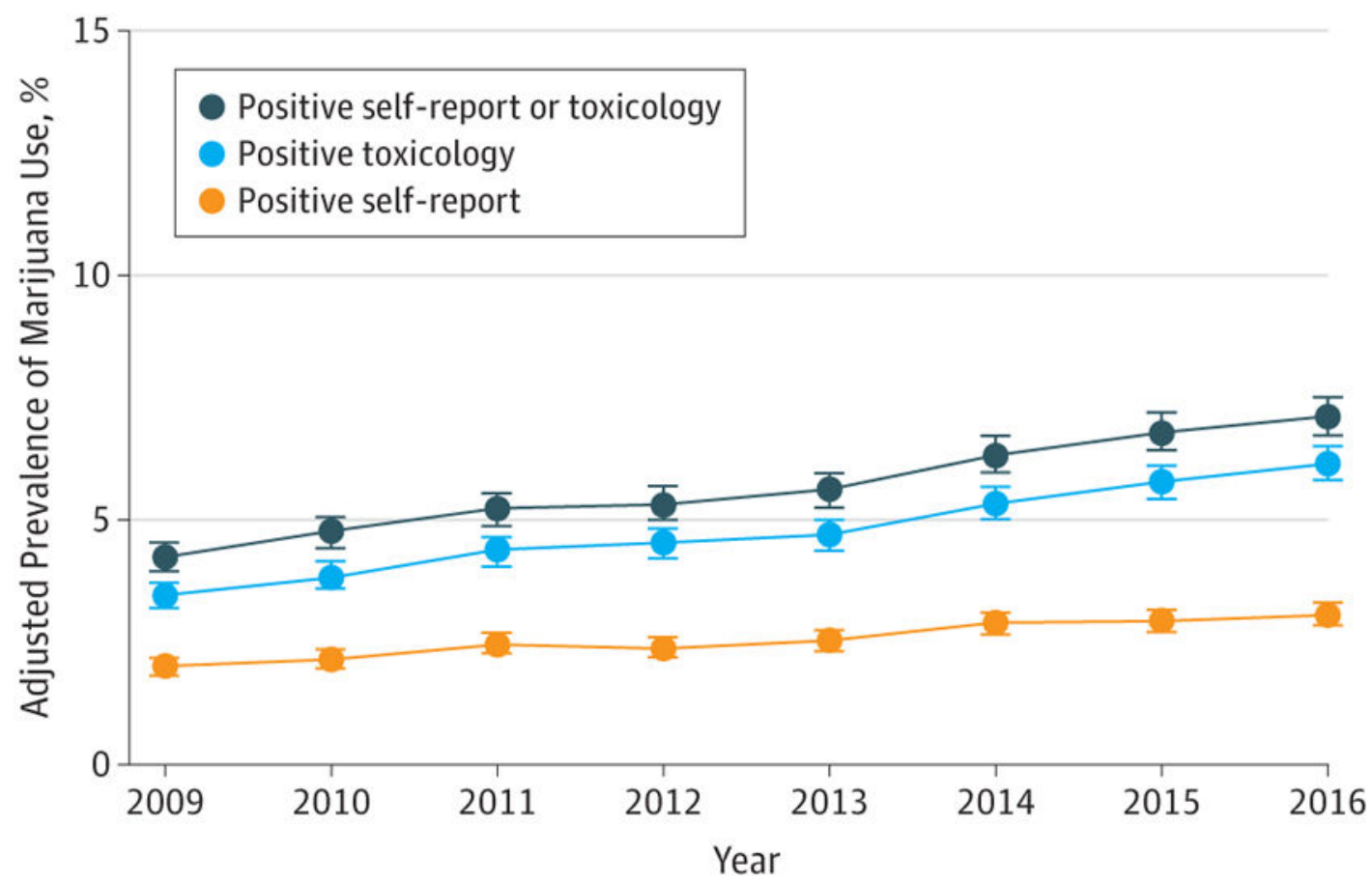

Figure 1. Adjusted Prevalence of Marijuana Use Among 279457 Pregnant Females in KPNC by Screening Type, 2009-2016

KPNC indicates Kaiser Permanente Northern California. Error bars indicate 95\% CIs of the adjusted prevalences. Poisson regression models controlled for age group, race/ethnicity, and median neighborhood household income (extracted from the electronic health record). The self-reported use questionnaire and toxicology screening were part of standard prenatal care (at approximately 8 weeks' gestation; 84\% of females completed both screenings during their first trimester). For toxicology tests, $58 \%$ were completed at the same visit as the selfreported use questionnaire, $87 \%$ were completed within 14 days of completing the questionnaire, and $100 \%$ were completed within 8 weeks of completing the questionnaire. All toxicology tests were confirmed with a confirmatory laboratory test. Among pregnant females with a positive test result for prenatal marijuana use, $15.9 \%$ were positive on selfreport only, $54.9 \%$ were positive on toxicology only, and $29.2 \%$ were positive on both selfreport and toxicology. The sample of females included in the study was generally representative of the population of all pregnant females. There were only small differences in the demographic characteristics of females who were included vs excluded (due to missing data on prenatal marijuana use), indicating minimal to no selection bias. The median sample size across years was 34322 (range, 32 813-38 919). 


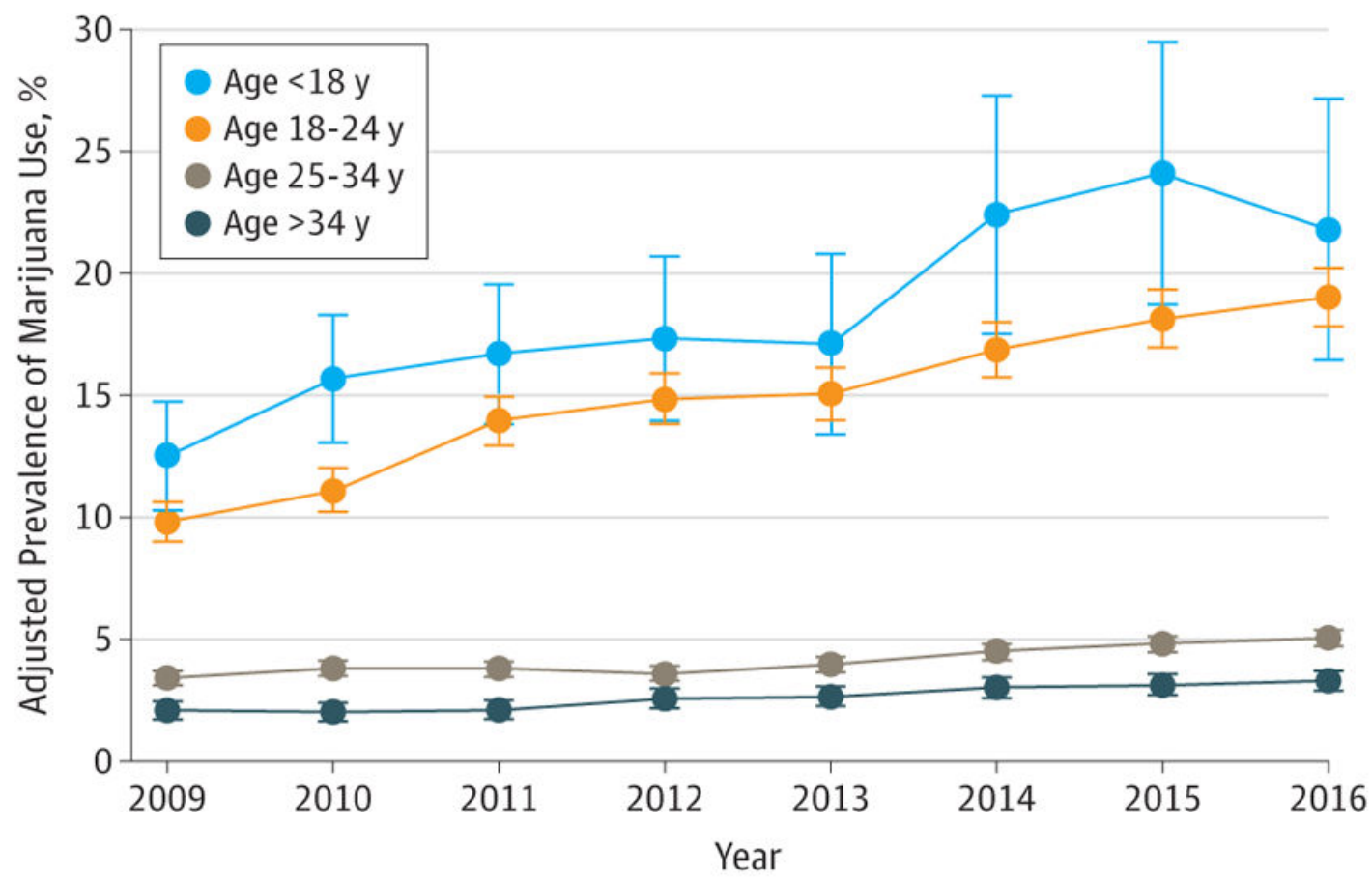

Figure 2. Adjusted Prevalence of Marijuana Use Among 279457 Pregnant Females in KPNC by Age, 2009-2016

KPNC indicates Kaiser Permanente Northern California. Results were based on positive self-report or positive toxicology screening. Error bars indicate $95 \%$ CIs of the adjusted prevalences. Age groups included younger than 18 years $(\mathrm{n}=3813 ; 1.4 \%) ; 18$ to 24 years $(\mathrm{n}$ $=44112 ; 15.8 \%) ; 25$ to 34 years $(n=172278 ; 61.6 \%)$; and older than 34 years $(n=59254$; $21.2 \%$ ). Poisson regression models by age group controlled for race/ethnicity and median neighborhood household income (extracted from the electronic health record). The selfreported use questionnaire and toxicology screening were part of standard prenatal care (at approximately 8 weeks' gestation; $84 \%$ of females completed both screenings during their first trimester). For toxicology tests, 58\%were completed at the same visit as the selfreported use questionnaire, $87 \%$ were completed within 14 days of completing the questionnaire, and $100 \%$ were completed within 8 weeks of completing the questionnaire. All toxicology tests were confirmed with a confirmatory laboratory test. Among pregnant females with a positive test result for prenatal marijuana use, $15.9 \%$ were positive on selfreport only, $54.9 \%$ were positive on toxicology only, and $29.2 \%$ were positive on both selfreport and toxicology. The sample of females included in the study was generally representative of the population of all pregnant females. There were only small differences in the demographic characteristics of females who were included vs excluded (due to missing data on prenatal marijuana use), indicating minimal to no selection bias. The median (range) sample size for each age group was 443 (236-779) for less than 18 years; 5485 (5221-5956) for 18 to 24 years; 21258 (20 002-24 347) for 25 to 34 years; and 7236 (6532-8848) for more than 34 years. 